\title{
BMJ Open Making shared decision-making (SDM) a reality: protocol of a large-scale long- term SDM implementation programme at a Northern German University Hospital
}

Marion Danner (D) , ${ }^{1}$ Friedemann Geiger, ${ }^{1,2}$ Kai Wehkamp, ${ }^{1,2}$ Jens Ulrich Rueffer, ${ }^{2,3}$ Christine Kuch, ${ }^{1}$ Leonie Sundmacher, ${ }^{4}$ Tove Skjelbakken, ${ }^{5}$ Anne Rummer, ${ }^{1}$ Anna Novelli (1) , ${ }^{4}$ Marie Debrouwere, ${ }^{1}$ Fueloep Scheibler, ${ }^{1,2}$ On behalf of the SHARE TO CARE (S2C) Project Team

To cite: Danner M, Geiger F, Wehkamp K, et al. Making shared decision-making (SDM) a reality: protocol of a large-scale long-term SDM implementation programme at a Northern German University Hospital. BMJ Open 2020;10:e037575. doi:10.1136/ bmjopen-2020-037575

- Prepublication history for this paper is available online. To view these files, please visit the journal online (http://dx.doi. org/10.1136/bmjopen-2020 037575).

Received 10 February 2020 Revised 28 June 2020 Accepted 21 September 2020

Check for updates

(c) Author(s) (or their employer(s)) 2020. Re-use permitted under CC BY-NC. No commercial re-use. See rights and permissions. Published by BMJ.

For numbered affiliations see end of article.

Correspondence to

Dr Marion Danner;

marion.danner@uksh.de

\section{ABSTRACT}

Introduction Shared decision-making (SDM) is not yet widely used when making decisions in German hospitals. Making SDM a reality is a complex task. It involves training healthcare professionals in SDM communication and enabling patients to actively participate in communication, in addition to providing sound, easy to understand information on treatment alternatives in the form of evidence-based patient decision aids (EbPDAs). This project funded by the German Innovation Fund aims at designing, implementing and evaluating a multicomponent, large-scale and integrative SDM programme-called SHARE TO CARE (S2C)—at all clinical departments of a University Hospital Campus in Northern Germany within a 4-year time period.

Methods and analysis S2C tackles the aforementioned components of SDM: (1) training physicians in SDM communication, (2) activating and empowering patients, (3) developing EbPDAs in the most common/relevant diseases and (4) training other healthcare professionals in SDM coaching. S2C is designed together with patients and providers. The physicians' training programme entails an online and an in situ training module. The decision coach training is based on a similar but less comprehensive approach. The development of online EbPDAs follows the International Patient Decision Aid Standards and includes written, graphical and video-based information. Validated outcomes of SDM implementation are measured in a preintervention and postintervention evaluation design. Process evaluation accompanies programme implementation. Health economic impact of the intervention is investigated using a propensity-score-matched approach based on potentially preference-sensitive hospital decisions. Ethics and dissemination Ethics committee review approval has been obtained from Medical Ethics Committee of the Medical Faculty of the ChristianAlbrechts-University Kiel. Project information and results will be disseminated at conferences, on project-hosted websites at University Hospital Medical Center Schleswig Holstein and by $\mathrm{S} 2 \mathrm{C}$ as well as in peer-reviewed and professional journals.
Strengths and limitations of this study

This study is the first large-scale long-term implementation of share decision-making (SDM) in an entire University Hospital involving all stakeholders in patient care in a multicomponent intervention.

Due to the size of our target intervention unit, a comparative study randomising comparable hospitals was neither feasible nor affordable.

- This study aims to detect important SDM implementation barriers and supporting factors in a busy and profit-oriented hospital setting.

- One limitation might be that there are no strong incentives for healthcare professionals and patients to contribute to the implementation of SDM.

- Another limitation is that no patients were involved in the design of this study.

\section{INTRODUCTION}

Shared decision-making (SDM) between healthcare professionals like physicians or nurses and patients is currently not a standard in German hospitals. ${ }^{12}$ SDM has rather been implemented sporadically in individual indications and healthcare settings. ${ }^{3}{ }^{4}$ This lack of SDM in routine settings might be due to a range of provider, patient, organisational, economic and contextual factors. ${ }^{2}{ }^{2}$ On the other hand, German legislation with the Patients' Rights Act gave SDM a more prominent role in German healthcare in 2013. ${ }^{6}$ The act implies that healthcare professionals and patients follow SDM communication rules. For example, physicians have to comprehensively inform their patients about relevant treatment alternatives $(\$ 630 \mathrm{e}){ }^{6}{ }^{6}$ In this context, the law points out that written material like patient decision aids may support 
professionals in meeting these legal requirements. While legislation in Germany hence seems to be ready for SDM and supporting instruments such as evidence-based patient decision aids (EbPDAs), stakeholders in daily practice are not yet routinely implementing it.

For SDM to be effective, the patients' and the healthcare providers' ability and willingness to participate in SDM are crucial. ${ }^{27}$ To make SDM a reality in any healthcare setting is an ambitious endeavour and a complex multilevel task. ${ }^{5}$ It involves training physicians and other healthcare professionals in SDM communication skills as well as encourage patients to actively participate in communication, in addition to providing evidence-based, easy to understand information on treatment alternatives to patients and their physicians. ${ }^{8}$ To be effective in daily practice, SDM should be codesigned with involved stakeholders to gain acceptance and recognition. ${ }^{3}$ In addition, it needs an inner (ie, within the institution that wants to do SDM) and an outer (concerning the external conditions in which the institution works) settings, in which programme implementation is possible, as defined, for example, by the Consolidated Framework for Implementation Research (CFIR) (see table 1 for this project). ${ }^{9}$ The Norwegian 'Decision Aid (DA) Factory' approach of the University Hospital North Norway, in which researchers and developers of SDM components-so-called 'knowledge producers'-work in close cooperation with the physicians and patients-so-called 'knowledge users'inspired implementation processes in this project. ${ }^{10}$

Individual components of SDM such as SDM training for healthcare professionals, patient activation/empowerment programmes or decision aids have all been previously tested in specific indications, populations and using different study designs. ${ }^{11-15}$ Their effectiveness and impact on decision processes have been assessed. For example, according to a recent systematic review of 115 randomised controlled trials (RCTs) with about 35000 patients altogether, the use of only EbPDAs to inform patients in specific indications led to improved health education/ literacy, more active participation and value congruent choices, more accurate expectations regarding course of disease and risk perceptions, more treatment satisfaction and better adherence to treatment. ${ }^{14}$ This finding has been reinforced by reviews in other specific populations. ${ }^{16}$ However, while most of the EbPDAs were previously tested in RCTs, they were often not subsequently used in the settings they were developed in. ${ }^{3}$ A recent study by Stacey et al? concluded that 'To improve subsequent use, researchers should codesign EbPDAs with end users to ensure fit with clinical practice and develop an implementation plan'. That study surveyed EbPDA developers who reported that the lack of physicians supporting and agreeing with the EbPDAs often hindered successful implementation. Training physicians in SDM in theory and practice has equally demonstrated to be effective, but the certainty of this evidence is low and limited to specific treatment settings. ${ }^{15}{ }^{17-19}$ While there may still be a lack of evidence regarding the effectiveness of SDM on patient-relevant clinical endpoints, there is growing agreement and consensus that SDM is a necessity, a patients' and a citizen's right, and an ethical imperative. ${ }^{7}$

It has also become clear that effectiveness to a large extent will depend on effective implementation strategies and consistent stakeholder involvement. ${ }^{35}$ Hence, given a growing body of evidence supporting the effectiveness of individual SDM interventions, the next step on the 'continuum of increasing evidence' according to Campbell $e t a l^{2021}$ would be to roll out the combined implementation of SDM interventions on a larger-scale in a long-term implementation study. Few programmes until now have addressed the simultaneous implementation of a range of SDM components at the same time (see eg, Sondergaard $e t a l^{22}$ Steffensen $e t a l^{23}$ ), some are currently ongoing (see eg, $\mathrm{Scholl}^{24}$ ), but none have yet introduced a multicomponent SDM programme at all departments of a hospital at a time. Therefore, in this publicly funded project, the objective was to design, implement and evaluate a multicomponent, large-scale and integrative SDM programme-called SHARE TO CARE (S2C) - at the University Hospital Medical Center Schleswig Holstein (UKSH), Campus Kiel, within a 4-year time period-from October 2017 until 30 September 2021. The project is designed and implemented in cooperation between the UKSH, Kiel, Germany and the University Hospital of Northern Norway, Troms $\varnothing$, Norway.

\section{METHODS AND ANALYSIS}

\section{Study design}

This study implies the large-scale implementation of SDM at the University Hospital Campus Kiel within a 4-year time period based on the S2C intervention programme. It includes comprehensive outcome evaluation with measurement of (1) SDM level in patient-physician interactions based on patients' and external observers' perceptions before and after S2C implementation and (2) measuring the impact of the S2C intervention on healthcare use and costs in comparison to a propensityscore-matched comparison population not exposed to S2C. The programme will be accompanied by a process evaluation based on the recommendations of the Medical Research Council Guidance and using the CFIR to guide development and implementation activities. ${ }^{925}$

The term 'multicomponent' in the S2C programme refers to four different interventions (components) designed and implemented simultaneously in several clinical departments. This includes (1) SDM training for physicians, ${ }^{17-19}{ }^{26}$ (2) SDM qualification as 'decision coach' for other healthcare professionals like nurses or physiotherapists, ${ }^{18} 27$ (3) the Ask Three Questions programme that aims at patient activation and empowerment and $^{28}{ }^{29}$ (4) development of online EbPDAs. ${ }^{14}$ These components and the respective responsible S2C project teams are depicted in figure 1 .

The term 'large-scale' means that the programme will sequentially be implemented at the University Hospital 


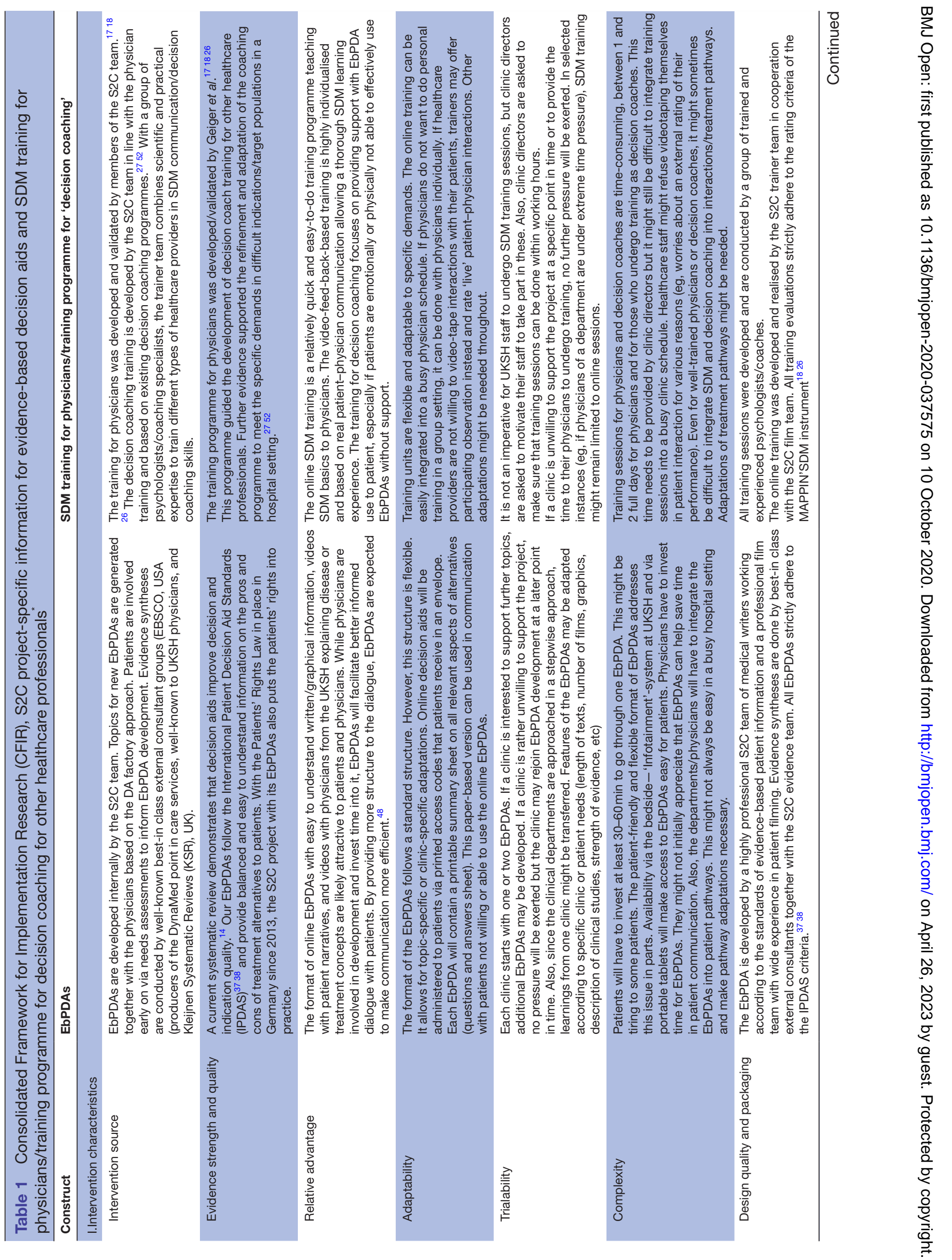




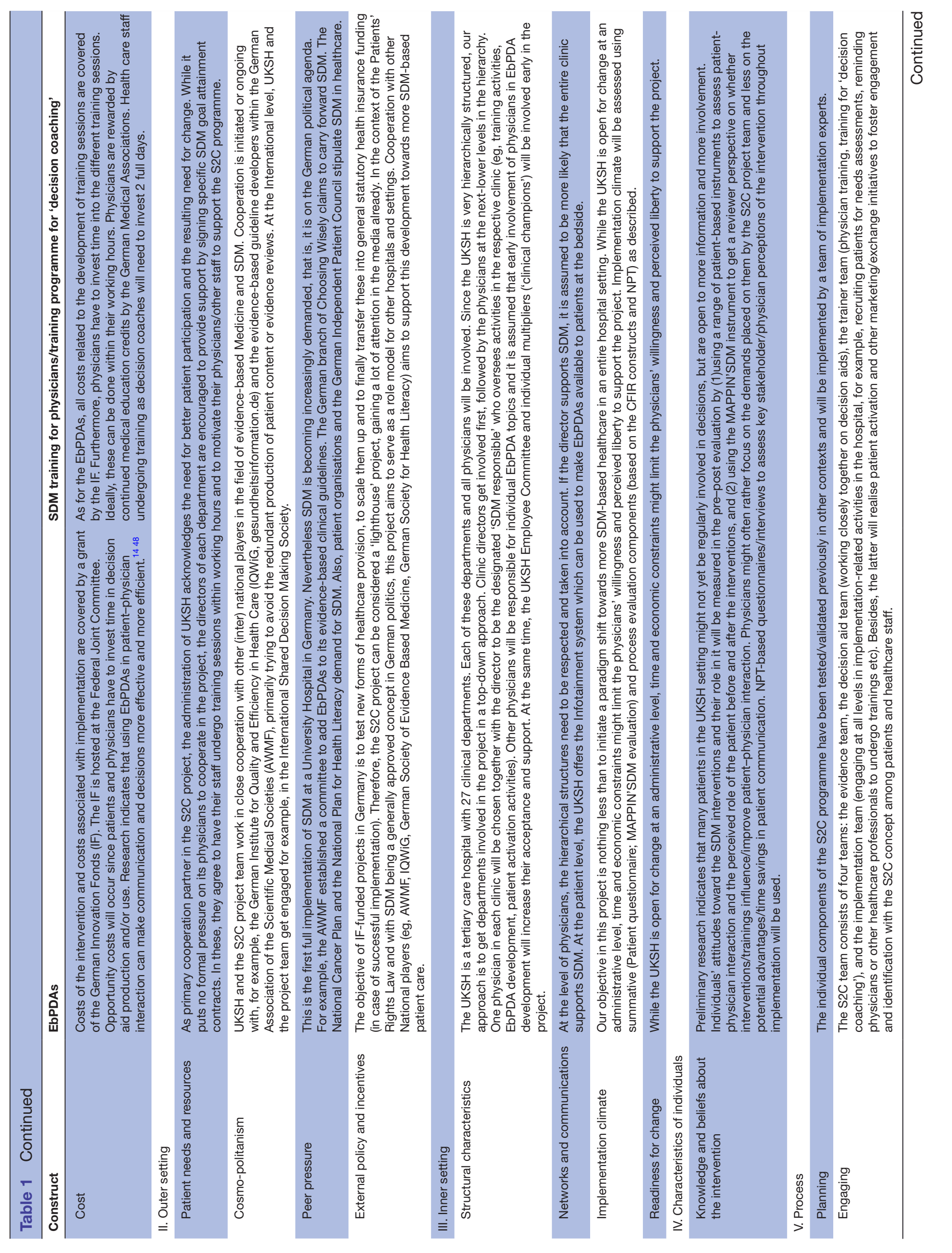




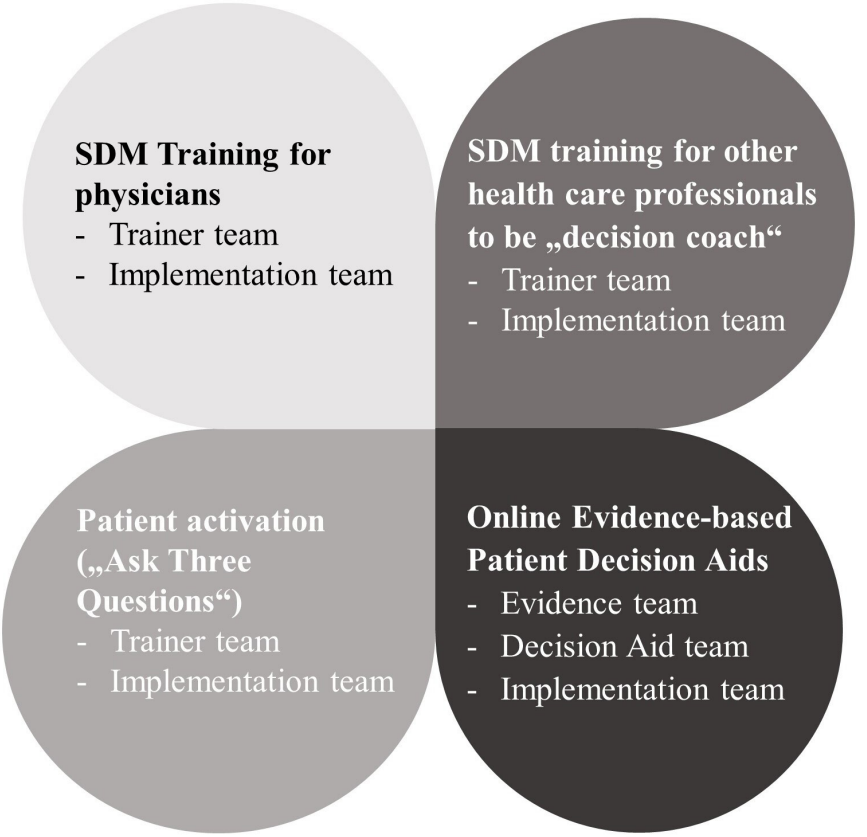

Figure 1 Project components and respective S2C project teams. S2C, SHARETO CARE, SDM, shared decisionmaking.

Campus Kiel involving 27 clinical departments with more than 650 physicians. The aim is to develop 83 EbPDAs enrolling new clinical departments into the programme every 6 months and identifying EbPDA topics at each clinic (figure 2). At the same time, each physician in the respective clinic undergoes SDM training. The Ask Three Questions patient activation is implemented simultaneously. In addition, in selected departments, a total of 150 other healthcare professionals will be trained as decision coaches to facilitate EbPDA use in specific patient target groups.

The term 'integrative' in S2C means that patients and healthcare professionals will be actively involved from the very beginning and throughout implementation, most actively not only in EbPDA development but also, for example, in training evaluation and in the patient activation programme. ${ }^{10}$ The integrative approach begins with identifying new topics together with physicians and conducting needs assessments with patients. It ends with having physicians distribute EbPDAs to patients in their clinical departments. Sample patients will also user test the EbPDAs before these will be administered to patients in daily practice.

\section{Patient and public involvement}

No patient was involved in the development or design of this study.

\section{Theoretical framework}

At the microlevel (level of healthcare professionals or patients), the S2C programme is designed and implemented on the grounds of the Theory of Planned Behavior suggesting behaviour is a result of motivation (intention) and ability (perceived behaviour control)..$^{30} 31$ 


\begin{tabular}{|lllllll}
\hline $\begin{array}{l}\text { Quarters } \Rightarrow \\
\text { Project phase } \Omega\end{array}$ & Q4/17-1/18 & Q2/18-Q3/18 & Q4/18-Q1/19 & Q2/19-Q3/19 & Q4/19-Q120 & Q2/20-Q3/20 \\
\hline
\end{tabular}

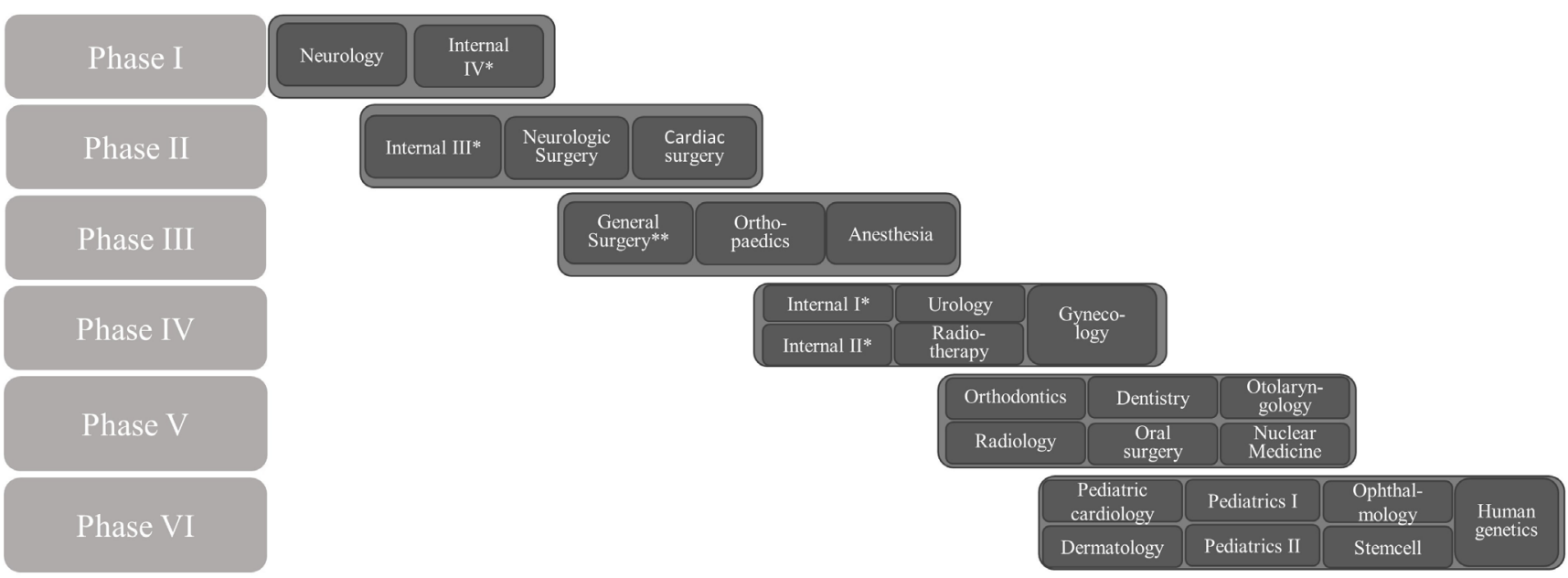

*Internal I: gastroenterology, hepatology, pneumology, internal intensive care medicine, endocrinology, infectiology, rheumatology, nutritional and ageing medicine; Internal II: hematology, oncology; Internal III: cardiology, angiology and internal intensive care medicine; Internal IV: renal and hypertensive diseases **General Surgery: visceral, thoracic, transplant and pediatric surgery

Figure 2 Sequential quarterly enrolment of new clinical departments.

Accordingly, the S2C programme aims to induce attitude and perception changes by training physicians and other healthcare professionals in SDM and by informing patients to enable simultaneous behaviour change at the level of patients and healthcare providers. The interactive process of EbPDA-development also aims at changing attitudes at the individual physician level. The implementation of the S2C programme is at the microlevel guided by the concept of normalisation process theory (NPT). The four components of the NPT are coherence (does the programme make sense to those who are involved?), participation (how do relevant stakeholders participate in implementation?), collective action (what to do to make implementation successful?) and reflexive monitoring (how do the involved individuals judge implementation processes?). ${ }^{32}$ As part of a process evaluation, these questions/constructs will be addressed with key stakeholders at specific points in time throughout the 4-year project time to continuously monitor implementation processes at the level of all involved stakeholders at the University Hospital Campus Kiel.

The complexity of this project taking into account context and processes of project implementation is depicted in table 1 following the CFIR (https://cfirguide.org/). This framework comprises five domains (intervention characteristics, outer setting, inner setting, characteristics of individuals and process) and 39 related constructs. ${ }^{9334}$ The constructs of the CFIR were used to describe the status quo of relevant project characteristics, project settings and potential interactions between these at project initiation. CFIR will also guide our implementation processes as described later.

\section{Setting and study population}

Campus Kiel as part of the UKSH Medical Center is a tertiary care hospital with more than 200000 cases treated each year. Twenty-seven clinical departments with more than 650 physicians and more than 150 other healthcare professionals and their patients will be part of either training modules or development and use of decision aids or both. New clinical departments and their patients will be sequentially enrolled in the study (figure 2 ).

\section{S2C intervention components}

Intervention 'SDM training for physicians'

This module aims at providing structured SDM training in three steps to a minimum of $80 \%$ of physicians working at the UKSH (ie, at least 520 physicians should receive training). The module is based on the pretested and validated training approach that has demonstrated to be effective and lead to an increased patient, physician and observer perception of involvement in decisionmaking. ${ }^{1718}$ Preceding training, each physician has to take a baseline video of him or herself with a patient in a real decision-making interaction. The physician then undergoes an online video tutorial that contains general information on SDM and its application in clinical practice. It also contains fictional interactions between physician and patient actors teaching physicians to differentiate 'good' from SDM communication 'in need of improvement'. For the subsequent video-based small group training sessions, the baseline video recording of a patient-physician interaction and a second recording (following online training) are rated by the S2C trainer team (see table 2 for additional information). In the subsequent group training, each physician receives video-based trainer 


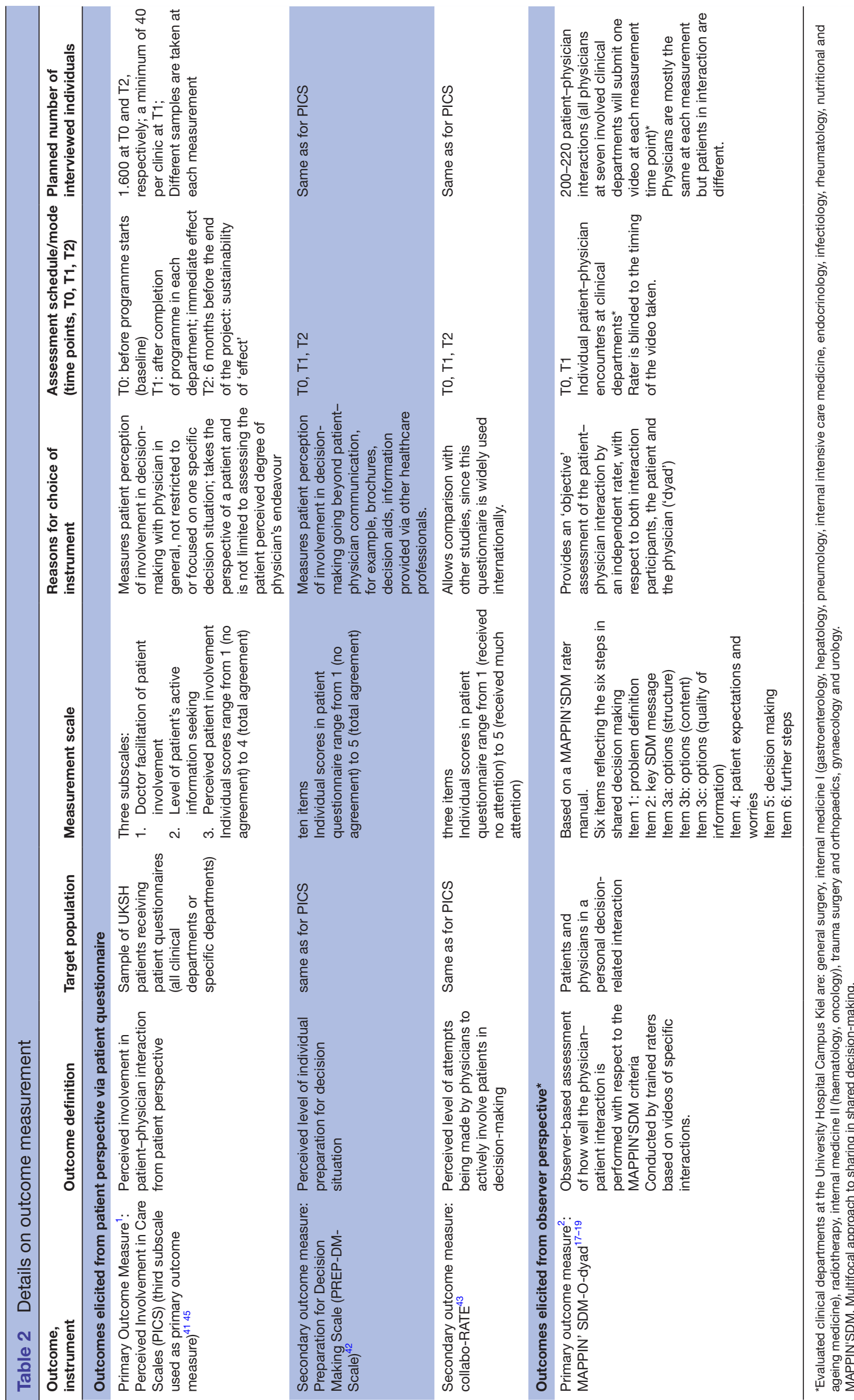


and group feedback. The aim is to provide an interactive and common SDM learning experience to physician. To increase their motivation, training participation is rewarded by continued medical education credits by the German Medical Associations.

\section{Intervention 'activation of patients'}

The 'Ask Three Questions' programme has originally been developed in Australia and tested in European countries. ${ }^{28}{ }^{29}$ Patients are instructed and motivated to actively participate in communication by asking their doctors questions regarding their specific (treatment) situation. Our patient activation concept communicates the message 'Ask Three questions-decide together' in a unique design at all departments using various distribution channels: paper postcards, posters/stand-up displays and screen-based messages inside UKSH. It will be embedded in several other interventions, like a patient homepage within the UKSH-homepage, the bedside infotainment system, information screens and special SDMdays in the central lobby.

\section{Intervention '(online) EbPDAs'}

Eigthy-three online EbPDAs will be developed, at least 1 in each department. The number is arbitrary, as there is no recommended number per department. We calculated the maximum possible number given the resources and the time frame of our grant. Consistent with the DA (decision aid) factory approach implementation starts with the identification of EbPDA topics together with physicians. Topics should be important for physicians, involve at least two preference-sensitive treatment alternatives and occur frequently. Topic specification with respect to target patient population, relevant treatment options and patient-relevant outcomes/issues of treatment is done based on a literature/guideline review and in exchange with physicians and patients. Needs assessments are conducted with about four to eight patients per topic to guide and structure EbPDAs as closely to patient needs as possible. Development of EbPDAs involves a systematic search and assessment of best available evidence for all relevant interventions, focusing on systematic reviews and evidence-based guidelines. Methods are based on the German standards of evidence-based patient information and the methods of evidence generation in patient information. ${ }^{35}{ }^{36}$ Text information on disease and treatment will be accompanied by video sequences with UKSH physicians and patients. In these sequences, physicians explain treatments and patients share their experience in decision-making. The latter is to motivate users of the online DAs to actively participate in decision-making. To avoid bias by testimonials, patients do not rate the different interventions in their video sequences but limit themselves to talking about their experience with the disease and their individual decision process. The process of DA development follows the International Patient Decision Aids Standards criteria (www.ipdas.ohri.ca ${ }^{37}{ }^{38}$ ). Each EbPDA undergoes external review.
Intervention 'SDM Training for other healthcare professionals to be decision coach'

This qualification module provides SDM training to about 150 nurses or other healthcare professionals in specific indications, where patients most likely will need support in using EbPDAs. Training principles are based on the physician training and decision coaching application in specific settings. ${ }^{12} 132739$ The goal is to train healthcare staff like nurses or physiotherapists to act as 'decision coaches' for their patients when using EbPDAs, that is, to simultaneously provide emotional, psychological and technical support. The qualification consists of 2 workshop days communicating the principles of SDM and EbPDAs and including two individual decision coaching sessions for each participant. In addition, each decision coach will be asked to videotape coaching communications with a patient two times and receive individual SDM trainer feedback. The communication between decision coaches and patients will always centre around a specific EbPDA.

\section{Study outcome and outcome measures}

The primary intervention outcome is whether and to what degree SDM-based interaction is provided to patients at UKSH. To cover different perspectives, we focus on two types of outcome measures, one providing the patient perspective and one providing an observer-based perspective (table 2). The primary outcome is based on a validated SDM measurement instrument, the Perceived Involvement in Care Scales (PICS) ${ }^{40}{ }^{41}$ It is a patientreported outcome instrument translated and validated in Germany and consists of three subscales with 4-5 items each. The subscales are (1) patient activation by doctors (five items) (2) active information-seeking behaviour (four items) and (3) perceived patient participation in decision-making (five items). Each item is measured on a scale from $1=$ 'do not agree at all' to $4=$ 'totally agree'. The second primary outcome consists in an observer rating of patient-physician interaction before and after the intervention using the MAPPIN'SDM (multifocal approach to sharing in shared decision making)-O(Observer)-dyad instrument. ${ }^{17} 1826$ MAPPIN'SDM-O-dyad measures the degree of SDM performance realised by the doctorpatient dyad (ie, by the unit made up of patient and physician) as rated by independent observers. The instrument consists of nine items assessing the process and quality of SDM. Each item is scored from ' 0 ' ('the indicator is not present') to ' 4 ' ('the indicator is present at an excellent standard'). The observer ratings are provided by independent but trained raters who rate video recordings of patient-physician interactions before and after the intervention (see 'data collection and analyses'). All observers are blinded to the measurement objects and time points of video recordings.

Additional secondary outcomes included in the patient questionnaire are two validated and widely used questionnaires, the Preparation for Decision Making Scale (PrepDM: 10 items; 5-point scale) ${ }^{42}$ and collaboRATE ${ }^{43}$ 


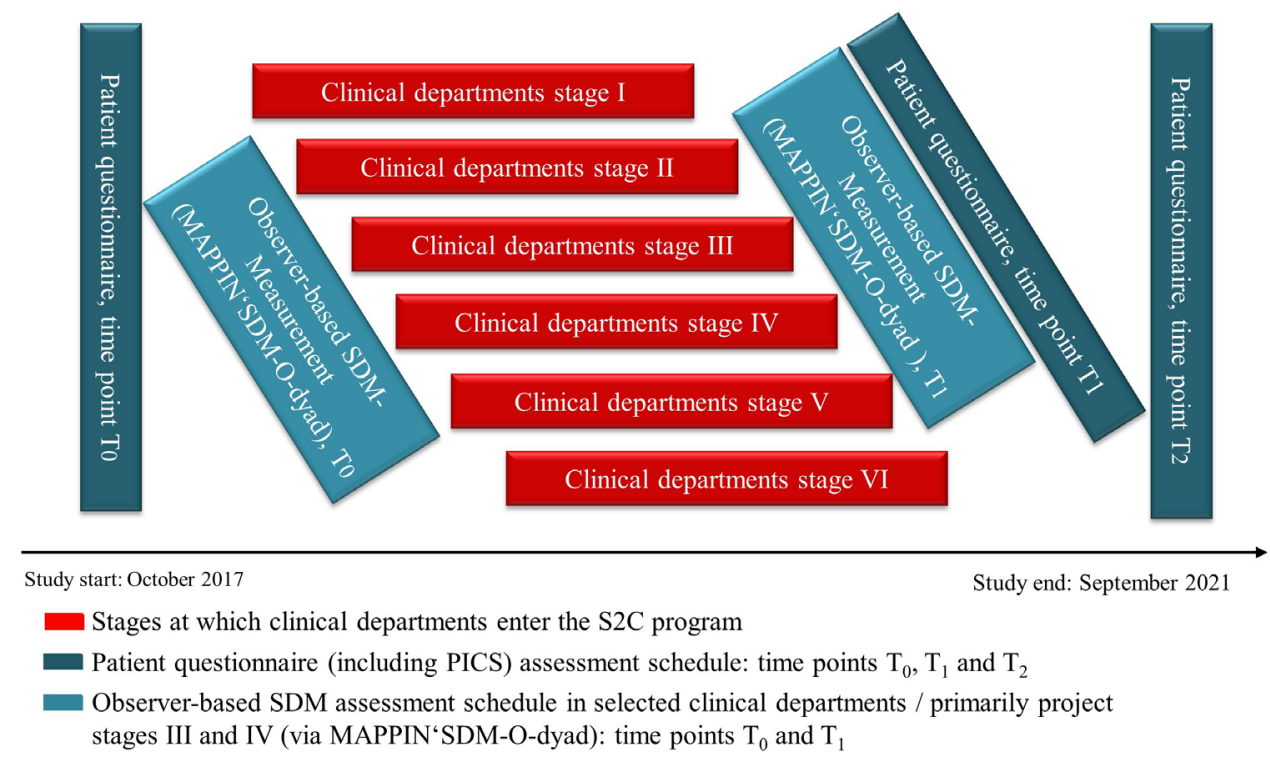

Figure 3 Project stages and data collection schedule for SDM evaluation. PICS, Perceived Involvement in Care Scales; S2C SHARE TO CARE; SDM, shared decision-making; MAPPIN'SDM, multifocal approach to sharing in shared decision-making.

(three items; 5-point scale). All outcome measures are detailed in table 2.

\section{Data collection}

Primary outcome data collection is conducted via patient questionnaire (including the PICS instrument) before $\left(\mathrm{T}_{0}\right)$ and two times after the intervention $\left(\mathrm{T}_{1}, \mathrm{~T}_{2}\right)$. The data collection and evaluation schedule are depicted in figure 3 .

The first patient questionnaire/PICS measurement $\left(\mathrm{T}_{0}\right)$ is scheduled at study initiation. The second $\left(\mathrm{T}_{1}\right)$ is taken after completion of the S2C intervention at each department to assess immediate intervention effect. The intervention is considered complete at the department level when at least $80 \%$ of physicians have undergone training, EbPDAs are developed and in use, and the patient activation programme is in place. The last measurement $\left(\mathrm{T}_{2}\right)$ is scheduled 6 months before study completion. It aims to appraise the sustainability of the S2C intervention. At $\mathrm{T}_{0}$ and $\mathrm{T}_{2}$, the patient questionnaire is mailed to a consecutive sample of patients who were hospitalised at the UKSH Kiel campus within the preceding weeks with a return envelope included in each mailing. At $T_{1}$, the questionnaire is sent to a respective sample of patients from a clinical department who completed the intervention. Patients who do not return the questionnaire within a 2-week or 4-week time frame, respectively, will get a reminder either one or two times. Based on the Total Design Method approach by Dillmann et al, ${ }^{44}$ final response rates of at least $60 \%-70 \%$ are expected.

The observer-based outcome measurement via MAPPIN'SDM-O-dyad is performed two times throughout the 4-year study period, at $\mathrm{T}_{0}$ and $\mathrm{T}_{1}$. To minimise workload for physicians, who must videotape encounters with patients to facilitate the MAPPIN'SDM-O-dyad evaluation, these assessments focus on central domains of hospital medicine (internal medicine, oncology, gynaecology, surgery, orthopaedics) being covered by specific clinical departments (departments of general surgery, internal medicine, radiotherapy, oncology \& haematology,gynaecology, trauma surgery \& orthopaedics, urology, gynaecology).

\section{Sample size calculation and data analyses}

Sample size calculation for the patient-based primary outcome is based on published PICS data. ${ }^{41}{ }^{45}$ An assumed difference of 0.4 in the PICS outcome at $\mathrm{T}_{1}$ versus $\mathrm{T}_{0}$ and a SD of 0.7 yields a sample size of about 40 for each clinical department at each measurement, using an independent sample $\mathrm{t}$ test and assuming a power of $80 \%$ and a level of significance of $5 \%$ (one-sided, assuming a positive effect of the SDM intervention). This yields a campuswide sample of 1080 patients (27 clinics, 40 patients per clinic). A difference in PICS scores of 0.4 comparing before and after measurement is considered relevant (Hedges $\mathrm{g}>0.5$, which corresponds to a medium size effect). If the distribution does not allow the assumption of normality, appropriate non-parametric tests will be applied in data analyses.

A presumed response rate of $60 \%-70 \%$ to the patient questionnaire mailings leads us to target about 1600 patients at measurement point $\mathrm{T}_{0}$ and $\mathrm{T}_{2}$ the campus level to finally achieve at least about 1000 patient questionnaires returned, yielding on average between 30 and 60 returned questionnaires per clinical department. These numbers will allow to measure significant differences in the primary endpoint not only at the campus but also at the individual department level (at least in the larger ones). At $\mathrm{T}_{1}$, a minimum of $65-70$ patients has to be contacted to have at least 40 questionnaires returned.

Sample size for the second primary outcome assessment (MAPPIN'SDM observer assessment) is given by the number of physicians at the involved clinical departments. Seven of the 27 UKSH departments will be part 
of the MAPPIN'SDM assessment. Physicians in these departments sum up to 200-220 in total. Each physician will deliver a patient-physician interaction video for outcome measurement at each measurement point. This analysis includes general surgery $(n=30-40$ physicians), internal medicine $(n=62)$, radiotherapy $(n=16)$, oncology/haematology $(n=21)$, orthopaedics $(n=27)$, gynaecology $(n=34)$ and urology $(n=10-20)$. Based on a previous study including training of physicians only, ${ }^{18}$ we aim at an effect size of $\mathrm{d}=0.5$ (Hedges $\mathrm{g}$ ). To yield a power of $80 \%$ (alpha=5\%), minimal sample size should be $\mathrm{n}=51$. Assuming a response rate above $60 \%(\mathrm{n} \geq 120)$, the sampling strategy leads to a sufficient sample size. It is hypothesised that in $80 \%$ of patient-physician interactions, patients will receive satisfactory SDM-based treatment at the second department-wide measurement $\left(\mathrm{T}_{1}\right)$ compared with less than $80 \%$ before the intervention $\left(\mathrm{T}_{0}\right)$. To answer the latter study hypothesis, a MAPPIN-SDM-Odyad mean value of greater or equal to 1.5 was defined as satisfactory basic patient involvement in decision-making based on previous validation research. ${ }^{18}$

\section{Health economic evaluation}

In addition to the pre SDM post SDM evaluation, an economic evaluation will be conducted. This analysis will be based on insurance claims data provided by the largest German Health Insurance provider (Techniker Krankenkasse (TK)). In Germany, approximately $88 \%$ of the population ( 72.8 million) is covered under the comprehensive statutory health insurance system. The TK provides health insurance for approximately 9.8 million people (13\% of the statutory contributors) and routinely collects data for reimbursement purposes on hospital stays, physician visits, medical procedures, medication and medical diagnoses. In the economic evaluation, incremental costs and use of specific services of patients admitted to the UKSH with preference-sensitive conditions in specific clinical departments (intervention group) will be compared with a matched population (control group) drawn from the administrative dataset from the TK. The control group includes patients with a hospital admission to another German University or Educational hospital (tertiary medical centre). From this sample population, patients will be matched to the intervention group using exact matching, propensity score matching or a combination of exact and propensity score matching. ${ }^{46}$ Matching criteria will include patient characteristics like age and sex, the main diagnosis of the hospital admission as well as measures of morbidity within 12 months preceding hospital admission. In line with previous research, ${ }^{48}$ variables that are compared across groups include preference-sensitive surgery rates, imaging rates, inpatient costs, total medical costs and hospital and emergency department admissions within 12 months after the admission to the hospital. To account for systematic differences between intervention and control group, the analysis will focus on the comparison of the difference in outcomes measured at two points in time, before and after the implementation of the SDM intervention. The analysis will be limited to about 10-15 frequently occurring and preference-sensitive conditions. These conditions will include but are not limited to cardiologic diseases, benign prostatic hyperplasia and other urologic diseases, benign uterine diseases and obstetrics, neurosurgery/back pain and orthopaedic diseases such as knee or hip replacement.

\section{Process evaluation}

Starting point for our evaluation is that the CFIR constructs as depicted in table 1. They summarise each study component, involved stakeholders, context (inner/ outer setting) and processes at study initiation. Each construct is followed up throughout the course of the study aiming to (1) identify areas where adaptations to initially planned implementation might be needed $\mathrm{and}^{2}$ (2) better understand which clinical departments might be more/less accessible to the SDM interventions and why. Process evaluation is done by using (a) documentation (eg, documentation of decision aid use by simply counting click/user numbers and times or documentation of number of physician trainings performed per clinical department) and (b) interview or structured questionnaire data. Interviews and structured questionnaires with stakeholders regarding implementation processes will be developed based on the described four concepts of the NPT theory. ${ }^{4-51}$ In addition, field notes are used by the respective project teams (figure 1) to adapt implementation strategies and processes to the specific demands of individual department's circumstances during the intervention phase.

\section{ETHICS AND DISSEMINATION}

The Medical Ethics Committee of the Medical Faculty of the Christian-Albrechts-University (CAU) Kiel has provided ethics approval to this study (reference number A111/18). This study will be conducted in accordance with German laws and regulations of the Medical Ethics Committee of the CAU, Kiel, Germany. Eligible patients or healthcare providers will be fully informed about the study and asked to participate in each part of the study: conducting personal interviews with patients (needs assessment), or video sequences with physicians/patients or involving physicians in training sessions. Patients/ providers will receive a respective information letter and will be informed about the implications of participation. They will have sufficient opportunity to ask questions and to consider the implications of the study before deciding to participate. Before participation, all individuals will provide written informed consent, compliant with the local and ethical data regulations. Patients and clinical staff will be allowed to withdraw from the study without giving a reason, at any time. The results arising from this implementation study will be presented at scientific meetings, on project-hosted websites at UKSH and by S2C as well as published in peer-reviewed journals. There is no 
intention to use professional writers and authorship will be based on the International Committee of Medical Journal Editors Guidelines.

\section{Author affiliations}

${ }^{1}$ SHARE TO CARE Team, University Medical Center Schleswig-Holstein Campus Kiel, Kiel, Germany

${ }^{2}$ SHARE TO CARE, Patientenzentrierte Kommunikation GmbH, Cologne, Germany

${ }^{3}$ TAKEPART Media \& Sciences GmbH, Cologne, Germany

${ }^{4}$ Ludwig-Maximilians-Universitat Munchen, Munchen, Germany

${ }^{5}$ Universitetet i Tromso Helsevitenskapelige fakultet Helsefak, Tromso, Norway

Acknowledgements The authors acknowledge Juergen Kasper and Katrin Liethmann for their tremendous contributions to the development of the study concept and intervention program.

Collaborators Corinna Knauff, Divna Tafelski, Johanna Gärtner, Stefanie Mevis, Heike Klein, Lea Kruse, Salim Greven, Gesine Steinbock, Kristina Blankenburg, Gerhard Koch, Claudia Hacke, Olga Kopeleva, Carmen Wiencke, Anja Schuldt, Christina Gesine Sommer, Barbara Kreidler, Constanze Stolz, Christine WagnerUllrich, Thorsten Duit, Michael Schipper, Lars Jacobsen, Christian Weymayr, Svenja Ludwig, Roya Shar-Yazdi, Ryan Naglatzki, Julia Bossert, Karoline Weik

Contributors FG, FS, KW and JUR developed the study concept and methods, designed the intervention program and are responsible for its implementation. LS and AN developed the evaluation concept and are responsible for its realisation. TS and CK provided substantial scientific and methodological contribution. AR and MDe provided methodological input and critically revised the manuscript. MD drafted the manuscript and provided scientific and methodological input to the study concept.

Funding This work was supported by a grant of the German Innovation Fonds (hosted by the Federal Joint Committee), grant number 01NVF17009.

Competing interests None declared.

Patient and public involvement Patients and/or the public were not involved in the design, or conduct, or reporting, or dissemination plans of this research.

Patient consent for publication Not required.

Provenance and peer review Not commissioned; externally peer reviewed.

Open access This is an open access article distributed in accordance with the Creative Commons Attribution Non Commercial (CC BY-NC 4.0) license, which permits others to distribute, remix, adapt, build upon this work non-commercially, and license their derivative works on different terms, provided the original work is properly cited, appropriate credit is given, any changes made indicated, and the use is non-commercial. See: http://creativecommons.org/licenses/by-nc/4.0/.

\section{ORCID iDs}

Marion Danner http://orcid.org/0000-0002-0653-4092

Anna Novelli http://orcid.org/0000-0002-4600-0183

\section{REFERENCES}

1 Wehkamp $\mathrm{KH}$, Naegler $\mathrm{H}$. The commercialization of patientrelated decision-making in hospital - a qualitative study of the perceptions of doctors and chief executive officers. Dtsch Ärztebl Int 2017;114:797-804.

2 Gemeinsam entscheiden im Klinikalltag: Ergebnisse von Fokusgruppengesprächen mit jungen Ärzten. Bertelsmann Stiftung, 2018. Available: file:///C:/SDM\%20Transfer/Publikation\%20SDM\%20 Projekt/Literatur/ Bittner_2018_VV_Studie_Gemeinsam_entscheiden_ final_online.pdf [Accessed 22 Jul 2020].

3 Stacey D, Suwalska V, Boland L, et al. Are patient decision AIDS used in clinical practice after rigorous evaluation? A survey of trial authors. Med Decis Making 2019;39:805-15.

4 Härter M, Dirmaier J, Scholl I, et al. The long way of implementing patient-centered care and shared decision making in Germany. Z Evid Fortbild Qual Gesundhwes 2017;123-124:46-51.

5 Tan ASL, Mazor KM, McDonald D, et al. Designing shared decisionmaking interventions for dissemination and Sustainment: can implementation science help translate shared decision making into routine practice? MDM Policy Pract 2018;3:2381468318808503.

6 Gesetz zur Verbesserung der Rechte von Patientinnen und Patienten (Law for the Improvement of Patients' Rights), 2013. Available: https://www.bundesaerztekammer.de/fileadmin/user_upload/ downloads/ Patientenrechtegesetz_BGBI.pdf [Accessed 22 Jul 2020].

7 Coulter A. Shared decision making: everyone wants it, so why isn't it happening? World Psychiatry 2017;16:117-8.

8 Maskrey N. Shared decision making: why the slow progress? An essay by Neal Maskrey. BMJ 2019;367:16762.

9 Damschroder LJ, Aron DC, Keith RE, et al. Fostering implementation of health services research findings into practice: a consolidated framework for advancing implementation science. Implement Sci 2009;4:50.

10 Kasper J, Lager AR, Rumpsfeld M, et al. Status report from Norway: implementation of patient involvement in Norwegian health care. $Z$ Evid Fortbild Qual Gesundhwes 2017;123-124:75-80.

11 Müller E, Strukava A, Scholl I, et al. Strategies to evaluate healthcare provider trainings in shared decision-making (SDM): a systematic review of evaluation studies. BMJ Open 2019;9:e026488.

12 Stacey D, Kryworuchko J, Bennett C, et al. Decision coaching to prepare patients for making health decisions: a systematic review of decision coaching in trials of patient decision AIDS. Med Decis Making 2012;32:E22-33.

13 Stacey D, Kryworuchko J, Belkora J, et al. Coaching and guidance with patient decision AIDS: a review of theoretical and empirical evidence. BMC Med Inform Decis Mak 2013;13:S11.

14 Stacey D, Légaré F, Lewis K, et al. Decision AIDS for people facing health treatment or screening decisions. Cochrane Database Syst Rev 2017;4:CD001431.

15 Légaré F, Adekpedjou R, Stacey D, et al. Interventions for increasing the use of shared decision making by healthcare professionals. Cochrane Database Syst Rev 2018;7:CD006732.

16 van Weert JCM, van Munster BC, Sanders R, et al. Decision AIDS to help older people make health decisions: a systematic review and meta-analysis. BMC Med Inform Decis Mak 2016;16:45.

17 Kasper J, Liethmann K, Heesen C, et al. Training doctors briefly and in situ to involve their patients in making medical decisionsPreliminary testing of a newly developed module. Health Expect 2017;20:1254-63.

18 Geiger F, Liethmann K, Reitz D, et al. Efficacy of the doktormitSDM training module in supporting shared decision making - Results from a multicenter double-blind randomized controlled trial. Patient Educ Couns 2017;100:2331-8.

19 Kienlin S, Kristiansen M, Ofstad E, et al. Validation of the Norwegian version of MAPPIN'SDM, an observation-based instrument to measure shared decision-making in clinical encounters. Patient Educ Couns 2017:100:534-41.

20 Campbell M, Fitzpatrick R, Haines A, et al. Framework for design and evaluation of complex interventions to improve health. BMJ 2000;321:694-6.

21 Campbell NC, Murray E, Darbyshire J, et al. Designing and evaluating complex interventions to improve health care. BMJ 2007;334:455-9.

22 Søndergaard SR, Madsen $\mathrm{PH}$, Hilberg O, et al. A prospective cohort study of shared decision making in lung cancer diagnostics: impact of using a patient decision aid. Patient Educ Couns 2019;102:1961-8.

23 Steffensen KD, Vinter M, Crüger D, et al. Lessons in integrating shared decision-making into cancer care. J Oncol Pract 2018;14:229-35.

24 Scholl I, Hahlweg P, Lindig A, et al. Evaluation of a program for routine implementation of shared decision-making in cancer care: study protocol of a stepped wedge cluster randomized trial. Implement Sci 2018;13:51.

25 Moore GF, Audrey S, Barker M, et al. Process evaluation of complex interventions: medical Research Council guidance. BMJ 2015;350:h1258.

26 Geiger F, Liethmann K, Hoffmann F, et al. Investigating a training supporting shared decision making (IT'S SDM 2011): study protocol for a randomized controlled trial. Trials 2011;12:232.

27 Berger-Höger B, Liethmann K, Mühlhauser I, et al. Informed shared decision-making supported by decision coaches for women with ductal carcinoma in situ: study protocol for a cluster randomized controlled trial. Trials 2015;16:452.

28 Shepherd HL, Barratt A, Jones A, et al. Can consumers learn to ask three questions to improve shared decision making? A feasibility study of the ASK (AskShareKnow) Patient-Clinician Communication Model $(囚)$ intervention in a primary health-care setting. Health Expect 2016;19:1160-8.

29 Shepherd HL, Barratt A, Trevena LJ, et al. Three questions that patients can ask to improve the quality of information physicians give about treatment options: a cross-over trial. Patient Educ Couns 2011;84:379-85

30 Ajzen I. The theory of planned behavior. Organ Behav Hum Decis Process 1991;50:179-211. 
31 Fishbein M. A reasoned action approach to health promotion. Med Decis Making 2008;28:834-44.

32 May CR, Finch T, Ballini L, et al. Evaluating complex interventions and health technologies using normalization process theory: development of a simplified approach and web-enabled toolkit. BMC Health Serv Res 2011;11:245.

33 Scalia P, Durand M-A, Forcino RC, et al. Implementation of the uterine fibroids option grid patient decision AIDS across five organizational settings: a randomized stepped-wedge study protocol. Implement Sci 2019;14:88.

34 Kirk MA, Kelley C, Yankey N, et al. A systematic review of the use of the consolidated framework for implementation research. Implement Sci 2016;11:72.

35 Leitlinie evidenzbasierte Gesundheitsinformation, 2017. Available: http://www.leitliniegesundheitsinformation.de/ [Accessed 22 Jul 2020].

36 General methods 5.0. IQWiG, 2017. Available: file:///C:/Users/ DANNER 1.TAK/AppData/Local/Temp/ General-Methods_Version-50.pdf [Accessed 24 May 2020].

37 Elwyn G, O'Connor A, Stacey D, et al. Developing a quality criteria framework for patient decision AIDS: online international Delphi consensus process. BMJ 2006;333:417.

38 Holmes-Rovner M. International Patient Decision Aid standards (IPDAS): beyond decision AIDS to usual design of patient education materials. Health Expect 2007;10:103-7.

39 Stacey D, Murray MA, Légaré F, et al. Decision coaching to support shared decision making: a framework, evidence, and implications for nursing practice, education, and policy. Worldviews Evid Based Nurs 2008:5:25-35.

40 Lerman CE, Brody DS, Caputo GC, et al. Patients' perceived involvement in care scale: relationship to attitudes about illness and medical care. J Gen Intern Med 1990;5:29-33.

41 Scheibler F, Freise D, Pfaff H. Die Einbeziehung von Patienten in die Behandlung - Validierung der deutschen PICS Skalen. Journal of Public Health 2004;12:199-209.

42 Bennett C, Graham ID, Kristjansson E, et al. Validation of a preparation for decision making scale. Patient Educ Couns 2010;78:130-3.
43 Forcino RC, Barr PJ, O'Malley AJ, et al. Using collaborate, a brief patient-reported measure of shared decision making: results from three clinical settings in the United States. Health Expect 2018;21:82-9.

44 Dillmann DA. Mail and Internet surveys. The tailored design method. New York: Wiley, 2007

45 Scheibler F, Pfaff H, Kowalski C, et al. Shared decision making in breast care centers in North Rhine-Westphalia: results of a 10-year trend analysis. Z Evid Fortb Qual Gesundhwes 2019;147-148:97-102.

46 Herold R, van den Berg N, Dörr M, et al. Telemedical care and monitoring for patients with chronic heart failure has a positive effect on survival. Health Serv Res 2018;53:532-55.

47 Ho DE, Imai K, King G, et al. Matching as nonparametric preprocessing for reducing model dependence in parametric causal inference. Political Analysis 2007;15:199-236.

48 Veroff D, Marr A, Wennberg DE. Enhanced support for shared decision making reduced costs of care for patients with preferencesensitive conditions. Health Aff 2013;32:285-93.

49 Rapley T, Girling M, Mair FS, et al. Improving the normalization of complex interventions: part 1 - development of the NoMAD instrument for assessing implementation work based on normalization process theory (NPT). BMC Med Res Methodol 2018;18:133

50 Finch TL, Girling M, May CR, et al. Improving the normalization of complex interventions: part 2 - validation of the NoMAD instrument for assessing implementation work based on normalization process theory (NPT). BMC Med Res Methodol 2018;18:135.

51 Finch TL, Rapley T, Girling M, et al. Improving the normalization of complex interventions: measure development based on normalization process theory (NoMAD): study protocol. Implement Sci 2013:8:43.

52 Rahn AC, Köpke S, Kasper J, et al. Evaluator-blinded trial evaluating nurse-led immunotherapy Decision Coaching In persons with relapsing-remitting Multiple Sclerosis (DECIMS) and accompanying process evaluation: study protocol for a cluster randomised controlled trial. Trials 2015;16:106. 